\title{
Efficacy of the Tent-Pole Technique in Horizontal Ridge Augmentation
}

\author{
Tu Lam Doan ${ }^{10}$, Lanh Duc Le ${ }^{1}(1)$
}

${ }^{1}$ Department of Dental Implantology, Faculty of Odonto-Stomatology, University of Medicine and Pharmacy, Ho Chi Minh City, Viet Nam.

Author to whom correspondence should be addressed: Tu Lam Doan, Department of Dental Implantology, Faculty of Odonto-Stomatology, University of Medicine and Pharmacy, Ho Chi Minh City, 652 Nguyen Trai Str., District 5, Ho Chi Minh City, Viet Nam. Phone: +84344053350. E-mail: 1amtubmt@gmail.com.

Academic Editor: Alessandro Leite Cavalcanti

Received: 10 March 2020 / Accepted: 03 June 2020 / Published: 15 June 2020

How to cite this article: Doan TL, Le LD. Efficacy of the tent-pole technique in horizontal ridge augmentation. Pesqui Bras Odontopediatria Clín Integr. 2020; 20:e5643. https://doi.org/10.1590/pboci.2020.109

\begin{abstract}
Objective: To evaluate the efficacy of the technique for ridge augmentation prior to implant placement. Material and Methods: Six patients with nine sites presenting an initial ridge width of smaller than $4 \mathrm{~mm}$ were included in this study. The tent-pole technique (combination of osteosynthesis screws measuring 1.5 $\mathrm{mm}$ in diameter, ABBM, and resorbable collagen membrane) was applied at defect sites. After eight months of healing time, implants were placed in the treated sites. The primary outcomes (radiographic initial ridge width, re-entry ridge width, ridge width gain) were measured by cone-beam computed tomography in reference buccopalatal cross-sections and the secondary outcomes (clinical parameters) were recorded by a digital caliper at the first and second stages. Results: After grafting, the radiographic ridge width increased by $3.02 \pm 1.11 \mathrm{~mm}(1.57-4.75 \mathrm{~mm})$ at $2 \mathrm{~mm}$ below the crest and $3.32 \pm 1.70 \mathrm{~mm}(0.23-5.66 \mathrm{~mm})$ at $6 \mathrm{~mm}$ below the crest significantly $(\mathrm{p}<0.05)$. For clinical results, the mean horizontal dimension gain was $3.21 \pm$ $1.04 \mathrm{~mm}(1.83-4.57 \mathrm{~mm})$, while the mean reduction in dimension was $0.38 \pm 0.33 \mathrm{~mm}$. These results were statistically significant $(\mathrm{p}<0.05)$. Uneventful healing and no infections or membrane exposure were recorded at all sites during the study process. Three of nine (33.33\%) defect sites required additional bone grafting. Conclusion: The tent-pole technique is an effective method for increasing the horizontal ridge dimension, minimizing postoperative complications, and facilitating subsequent implant placement.
\end{abstract}

Keywords: Dental Implantation; Dental Implants; Alveolar Process; Guided Tissue Regeneration. 


\section{Introduction}

Insufficient alveolar ridge width after tooth loss still leads to challenges in different clinical situations. Particularly, according to Len Tolstunov's edentulous ridge classification, severe defect areas (2-4 mm residual ridge width) should undergo reconstruction prior to implant placement [1]. Adequate bone volume permits proper implant position favorable to restorations and leads to optimized function and aesthetic outcomes consequently. A variety of reconstructive approaches have been introduced in order to obtain adequate bone volume prior to implant placement such as guided bone regeneration (GBR) [2], ridge split/expansion [3], block grafting [4], and distraction osteogenesis [5]. Ridge splitting may lead to buccal bone wall fractures, which negatively affect primary implant stability and the implant axis in some situations. Autogenous block grafting is associated with morbidity of donor sites, resorption of materials, and increased treatment costs [6].

GBR has demonstrated predictable results in lateral augmentation and long-term implant success. To avoid shrinkage resulting from pressure after flap closure, several space-maintenance modalities were used to facilitate reconstruct ridge formation such as tenting screws [7], titanium (Ti) meshes [8], and Ti-reinforced polytetrafluoroethylene (PTFE) membranes [9]. Nonresorbable membranes have a high risk of exposure, necessitating removal in the re-entry stage [9]. Resorbable membranes have shown better biocompatibility and support of underneath bone growth [10]. However, their lack of stiffness can lead to decreased bone volume gain. Therefore, the tent-pole technique, which combines resorbable collagen membrane and tenting screws, represents an effective approach, offering easy manipulation and low complication rates.

Some authors previously performed a randomized clinical trial comparing allograft alone to allograft mixed (1:1) particulate autogenous bone using a tenting screw technique [11]. The overall mean amount of ridge width gain was approximately $3 \mathrm{~mm}$ and the authors concluded that the tent-pole technique is a successful and predictable approach for regenerating atrophic ridges. Elsewhere, a retrospective survey was conducted comparing Ti-reinforced PTFE membrane, the tunnel technique, and tenting screws [9]. The results indicated that the use of tenting screws prompted a lower rate of complications relative to the PTFE membrane and may be more favorable than the tunnel technique in cases in which the flat bony is deficient [9].

Anorganic bovine bone mineral (ABBM) may be a suitable bone substitute material for adoption in staged lateral bone augmentation without the addition of particulate autogenous bone [6]. Previous authors concluded that using ABBM (Bio-Oss) combined with native collagen membrane was an effective treatment for horizontal bone augmentation [Q] .

To our knowledge, the use of ABBM as a unique grafting material in combination with collagen membrane in the tent-pole technique for horizontal ridge augmentation has not yet been investigated retrospectively. Therefore, this clinical study was conducted to evaluate the efficacy of the tenting screw technique in facilitating optimal regeneration ridge dimensions by measuring clinical and radiographic parameters pre- and at eight months of healing time postoperatively.

\section{Material and Methods}

Study Design and Sample

A clinical study was carried out. Six patients presenting nine maxilla defect sites that required horizontal bone augmentation prior to implant insertion were recruited into this study from April 2018 through April 2019 at the Department of Dental Implantology, University of Medicine and Pharmacy (UMP).

Inclusion criteria included (1) initial ridge width of $4 \mathrm{~mm}$ or less at $2 \mathrm{~mm}$ apical from the crest of the alveolar bone as assessed by using cone-beam computed tomography (CBCT) and (2) bone height allowing the 
insertion of at least $8 \mathrm{~mm}$ of implant length without the need for vertical augmentation. Patients were excluded if (1) they had an uncontrolled systematic condition that may affect the outcome of surgical treatment such as diabetes, bone metabolic disease, pregnancy, smoking, periodontitis, or oral infection or (2) if they had an insufficient vertical and horizontal combined ridge dimension.

\section{Surgical Protocol and Clinical Measurements}

\section{First Stage}

All patients were evaluated by a preoperatively biochemical blood test. Patients were prescribed amoxicillin $0.5 \mathrm{~g}$ (three times/day) for five days and ibuprofen 0.4 $\mathrm{g}$ (three times/day) three days following surgery and operation sites were disinfected by rinsing with $0.12 \%$ chlorhexidine gluconate solution for one minute. Local anesthesia was applied by lidocaine $2 \%$ with epinephrine 1:100,000.

A trapezoidal or triangular flap was made and advanced at least one adjacent tooth away from the defect site by using a no. 15 scalpel. This flap design facilitated tension-free primary closure. Consequently, a mucoperiosteal flap was reflected by using elevators. All soft-tissue remnants on bony surfaces were removed using large carbide round burs. The initial ridge width was measured by a digital caliper in the center of the defect site. In multiple continuous tooth loss situations, we measured the position of each tooth defined by the distance from the adjacent tooth to the center point of the wax-up tooth mesiodistal mount cast made previously.

After that, the first $1.5-\mathrm{mm}$ diameter titanium screw was placed slightly coronal inclined to the alveolar ridge at $3 \mathrm{~mm}$ below the crest, while a second one was placed at 4 to $6 \mathrm{~mm}$ away from the crest apically per each tooth loss site. Screw placement was completed until the attempted ridge width was obtained. The postoperative ridge width dimension was measured from the palatal bone surface to the screw head by a digital caliper. Small round burs were applied to decorticate the buccal wall. Following periosteal-releasing incisions, a native collagen membrane (Lyoplant ${ }^{\circledR}$; B. Braun, Melsungen, Germany) was adapted to completely cover the defect areas; the particulate ABBM (Geistlich Bio-Oss; Geistlich Pharma, Wolksen, Switzerland) was then placed around screws to the level of the screw head, and the membrane was placed to cover fully graft sites. Next, 4-O polyamide interrupted sutures were used to establish a primary closure flap without incorporating horizontal mattress sutures (Figure 1). All procedures were performed by only one surgeon.

Patients were instructed to take the prescribed medications; maintain oral hygiene; return for suture removal after 10 days; visit each month for graft site observation, and report postoperative complications such as membrane exposure, infection, or screw head perforation.

\section{Second Stage}

After eight months of healing, following observation of the full-thickness flap, tenting screws were removed. Measurement of re-entry alveolar ridge width and inserted implants were performed. Additional bone grafting was conducted simultaneously with implant placement if an insufficient horizontal ridge dimension was noted.

\section{Radiographic Measurement}

Before taking CBCT scans, a customized radiographic guide template was fabricated by self-curing acrylic with a homogeneous $2 \mathrm{~mm}$ of thickness and small holes containing Gutta-percha in the outside buccal and palatal surfaces. Adjustments to align with the reference buccopalatal cross-section were made through the 
radiopaque Gutta-percha points during the study process. In this cross-section, the initial ridge width and the ridge width gain at eight months after bone graft at $2 \mathrm{~mm}$ and $6 \mathrm{~mm}$ below the crest were measured perpendicularly to the implant axis (Figure 2). All clinical and radiographic parameters were measured by only one investigator.

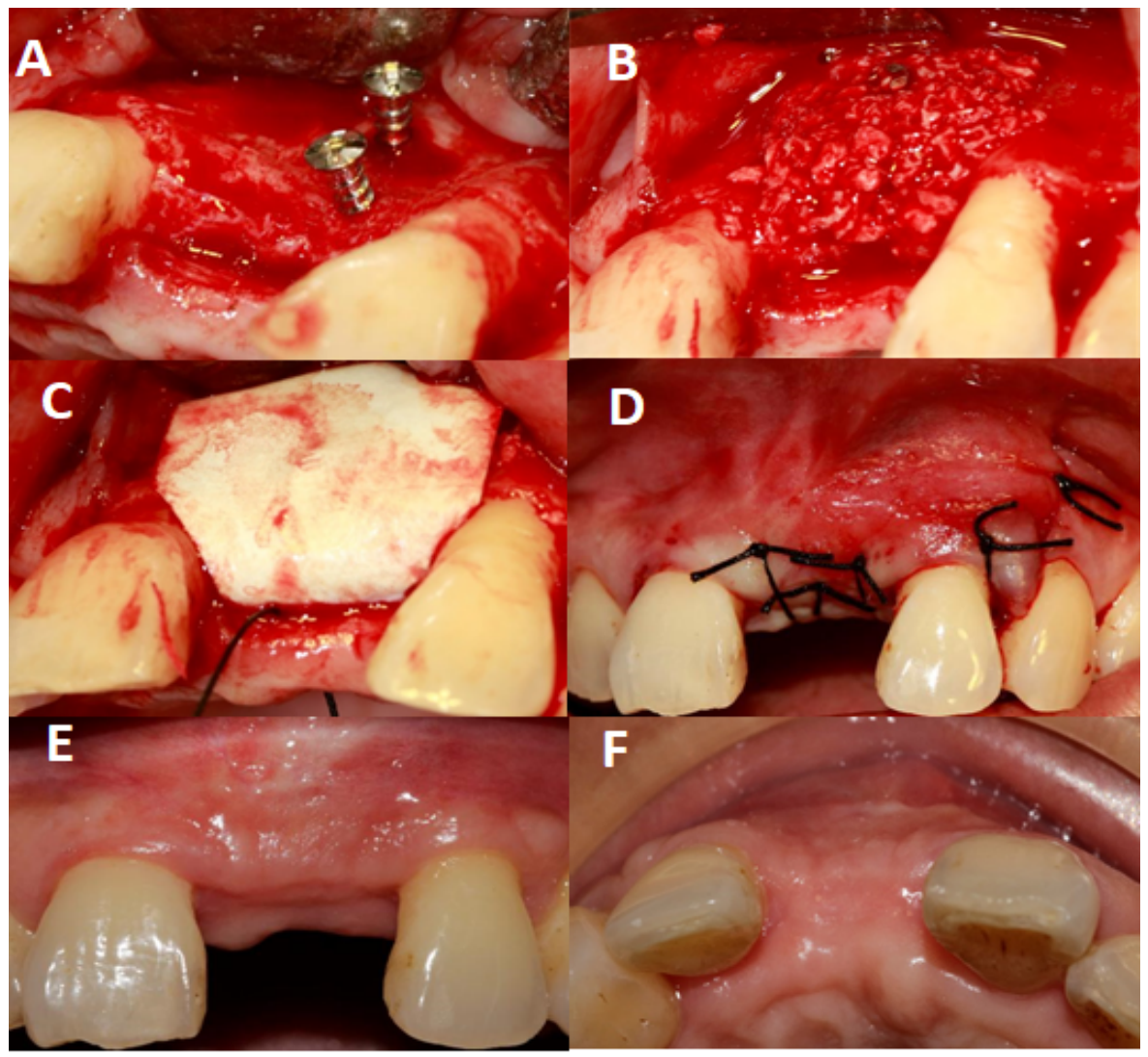

Figure 1. Step-by-step surgical protocol. A: Placement of tenting screws. B: Placement of graft materials. C: Membrane coverage. D: Suturing. E: After eight months of follow-up (frontal view). F: After eight months of follow-up (occlusal view).

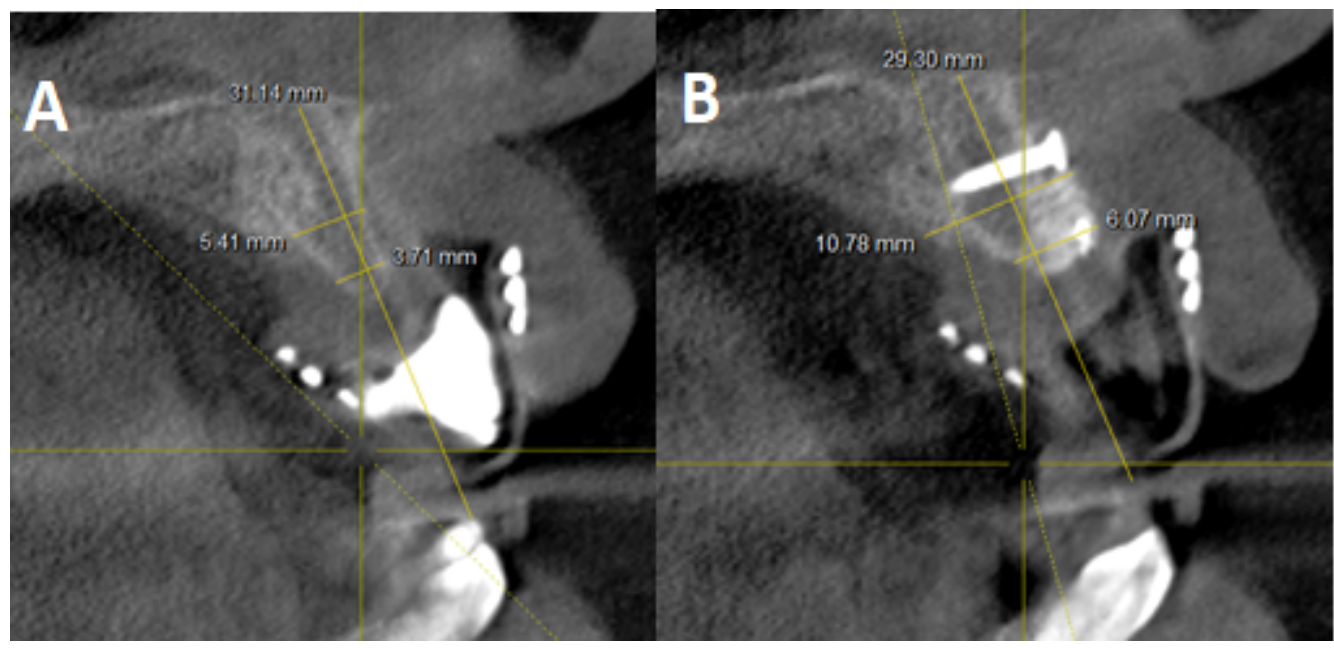

Figure 2. Radiographic measurement on reference buccopalatal cross-sections. A: Preoperative. B: Postoperative. 
Statistical Analysis

All clinical and radiographic data were statistically analyzed using the Stata 13.0 software program (Stata Corporation, College Station, TX, USA). The primary outcome was the radiographic ridge width gain at eight months and the secondary outcomes were clinical ridge width gain and postoperative complications. The Wilcoxon signed-rank and Mann-Whitney U tests were performed, with the significance level set at $5 \%$.

Ethical Aspects

Ethical approval was obtained from the ethical committee of University of Medicine and Pharmacy (Protocol No. 263/DHYD-HDDD).

\section{Results}

Six patients (two males and four females) were enrolled in the study with a mean age of 54.33 years (range: 43-62 years). A total of nine maxilla defect sites (five incisor and four premolar areas), including seven areas of single tooth loss and one area of dual tooth loss, were included. Of all the sites, there were two postextraction sites and seven edentulous sites with one $\mathrm{C}$ shape of ridge morphology and six flat shape ridges. Uneventful healing at all sites, no early or late membrane exposure, and no postoperative infections were recorded during the study process. Nine implants measuring 3.3 to $4.1 \mathrm{~mm}$ in diameter and 8 to $10 \mathrm{~mm}$ in length were successfully inserted into graft areas with good primary implant stability (>30 Ncm). Three sites $(33.33 \%)$ required additional bone grafting simultaneous with implant placement due to insufficient horizontal ridge dimensions. All sites accepted implant placement in the second stage without regrafting being necessary.

\section{Radiographic Measurement}

Overall, the tent-pole technique facilitated an average ridge width gain of $3.02 \pm 1.11 \mathrm{~mm}$ at eight months postoperation $(0.23-5.66 \mathrm{~mm})$ at a level $2 \mathrm{~mm}$ and $3.32 \pm 1.70 \mathrm{~mm}(0.23-5.66 \mathrm{~mm})$ at a level $6 \mathrm{~mm}$ below the alveolar crest. These results were significantly different $(\mathrm{p}<0.05)$. The initial ridge width was $3.10 \pm$ $0.65 \mathrm{~mm}(2.1-4.0 \mathrm{~mm})$ and the re-entry ridge width was $6.12 \pm 1.08 \mathrm{~mm}(4.93-7.95 \mathrm{~mm})$ at $2 \mathrm{~mm}$ away from the crest. The ridge width gain was not affected by site-relative factors such as post-extracted site/edentulous sites $(p=0.77)$, sex $(p=0.56)$, ridge morphology $(p=0.32)$, or tooth position $(p=0.81)$, but was affected by singleor multitooth loss $(\mathrm{p}<0.05$; Mann-Whitney $\mathrm{U}$ test).

Table 1 Radiographic measurement results.

\begin{tabular}{|c|c|c|c|c|c|c|c|}
\hline \multirow[b]{2}{*}{ Patient } & \multirow{2}{*}{$\begin{array}{c}\text { Tooth } \\
\text { Loss Sites }\end{array}$} & \multicolumn{2}{|c|}{$\begin{array}{l}\text { Initial Ridge Width } \\
(\mathrm{mm})\end{array}$} & \multicolumn{2}{|c|}{$\begin{array}{c}\text { Re-Entry Ridge Width } \\
(\mathrm{mm})\end{array}$} & \multicolumn{2}{|c|}{$\begin{array}{c}\text { Ridge Width Gain } \\
(\mathrm{mm})\end{array}$} \\
\hline & & at $2 \mathrm{~mm}$ & at $6 \mathrm{~mm}$ & at $2 \mathrm{~mm}$ & at $6 \mathrm{~mm}$ & at $2 \mathrm{~mm}$ & at $6 \mathrm{~mm}$ \\
\hline 1 & 24 & 3.36 & 5.39 & 4.93 & 5.62 & 1.57 & 0.23 \\
\hline 2 & 21 & 3.71 & 5.41 & 6.07 & 10.78 & 2.36 & 5.37 \\
\hline 3 & 12 & 2.10 & 6.46 & 4.99 & 8.62 & 2.89 & 2.16 \\
\hline 4 & 21 & 2.32 & 5.83 & 5.86 & 9.49 & 3.54 & 3.66 \\
\hline 5 & 11 & 3.21 & 4.21 & 5.20 & 7.36 & 1.99 & 3.15 \\
\hline \multirow[t]{4}{*}{6} & 14 & 4.00 & 5.00 & 6.04 & 8.91 & 2.04 & 3.91 \\
\hline & 21 & 2.50 & 3.40 & 6.47 & 9.06 & 3.97 & 5.66 \\
\hline & 24 & 3.20 & 6.20 & 7.95 & 10.03 & 4.75 & 3.83 \\
\hline & 25 & 3.50 & 8.10 & 7.59 & 10.04 & 4.09 & 1.94 \\
\hline \multicolumn{2}{|c|}{ Mean (SD) } & \multicolumn{2}{|c|}{$3.10 \pm 0.65$} & \multicolumn{2}{|c|}{$6.12 \pm 1.08$} & \multicolumn{2}{|c|}{$3.02 \pm 1.11$} \\
\hline \multicolumn{2}{|c|}{ p-value } & & & & & \multicolumn{2}{|c|}{$<0.05^{*}$} \\
\hline
\end{tabular}

*Wilcoxon signed-rank test. 
Clinical Measurement

At re-entry, the horizontal bone dimension significantly increased by $3.21 \pm 1.04 \mathrm{~mm}(1.83-4.57 \mathrm{~mm})$ and the mean bone resorption observed was $0.38 \pm 0.33 \mathrm{~mm}$, which were statistically significant $(\mathrm{p}<0.05)$.

Table 2. Clinical measurement results.

\begin{tabular}{|c|c|c|c|c|c|c|}
\hline Patient & $\begin{array}{l}\text { Tooth } \\
\text { Loss } \\
\text { Sites }\end{array}$ & $\begin{array}{c}\text { Initial Ridge } \\
\text { Width } \\
(\mathrm{mm})\end{array}$ & $\begin{array}{c}\text { Post-Op } \\
\text { Ridge Width } \\
(\mathrm{mm})\end{array}$ & $\begin{array}{c}\text { Re-Entry } \\
\text { Ridge Width } \\
(\mathrm{mm})\end{array}$ & $\begin{array}{c}\text { Reduced } \\
\text { Dimensions } \\
(\mathrm{mm})\end{array}$ & $\begin{array}{c}\text { Ridge Width } \\
\text { Gain } \\
(\mathrm{mm})\end{array}$ \\
\hline 1 & 24 & 3.24 & 5.74 & 5.07 & 0.67 & 1.83 \\
\hline 2 & 21 & 2.98 & 6.23 & 6.13 & 0.1 & 3.15 \\
\hline 3 & 12 & 2.06 & 5.22 & 4.87 & 0.35 & 2.81 \\
\hline 4 & 21 & 2.13 & 5.91 & 4.8 & 1.11 & 2.67 \\
\hline 5 & 11 & 3.16 & 6.13 & 6.07 & 0.06 & 2.91 \\
\hline \multirow[t]{4}{*}{6} & 14 & 3.91 & 6.43 & 5.98 & 0.45 & 2.07 \\
\hline & 21 & 1.98 & 6.63 & 6.54 & 0.09 & 4.56 \\
\hline & 24 & 3.16 & 7.98 & 7.73 & 0.25 & 4.57 \\
\hline & 25 & 3.15 & 7.82 & 7.45 & 0.37 & 4.3 \\
\hline \multicolumn{2}{|c|}{ Mean (SD) } & $2.86 \pm 0.66$ & $6.45 \pm 0.92$ & $6.07 \pm 1.06$ & $0.38 \pm 0.33$ & $3.21 \pm 1.04$ \\
\hline \multicolumn{2}{|c|}{ p-value } & & & & $<0.05^{*}$ & $<0.05^{*}$ \\
\hline
\end{tabular}

*Wilcoxon signed-rank test.

\section{Discussion}

The present study considered horizontal bone augmentation performed in the maxilla prior to implant placement by using tenting screws, xenograft materials, and resorbable membrane. In this study, although the clinical mean ridge width was measured at $2 \mathrm{~mm}$ below the crest, the result of ridge width gain was better than that in a similar study performed $(3.21 \mathrm{~mm}$ vs. $2.92 \mathrm{~mm})$. These authors conducted measurements at $3 \mathrm{~mm}$ away from the crest. Generally, the more apically bone graft position, the more ridge width gain. With three infected sites excluded, the mean bone gain was $3.22 \mathrm{~mm}$, similar to our results [11].

Our mean horizontal bone gain was smaller than that obtained by other authors, $3.6 \mathrm{~mm}[2]$ and 5.68 $\mathrm{mm}$ [12]; these authors used collagen membrane and ABBM but did not use tenting crews as spacemaintainers. A better outcome may be performing advanced flap and using bone tack to obtain good membrane stability [12]. The re-entry ridge width was $6.12 \mathrm{~mm}$ and not affected by ridge morphology, sex, postextraction site/edentulous site, or tooth loss area. These results were similar to previously reported [11] and demonstrated that the tent-pole technique was an effective approach for obtaining a sufficient horizontal ridge dimension in any edentulous areas. In all cases, the implants were inserted in the second stage without regrafting. The re-entry ridge width was affected by single or multiple tooth loss sites. In the context of multiple tooth loss sites, more significant horizontal bone gain was observed. This finding may be the result of the small sample size in this case series.

Using slowly absorbable material and tenting screws as space-maintaining elements may help to obtain a slightly reduced horizontal dimension of $0.38 \mathrm{~mm}(5.94 \%)$. According to a recent systematic review that investigated the stability of grafting material between augmentation procedures, the estimated mean bone resorption of GBR was $1.22 \mathrm{~mm}$ [13]. However, this information was extracted from previous studies that did not use the tent-pole technique. In clinical practice, at re-entry after eight months, we observed that new bone formation achieved the level of the head of the screws in almost all cases or even covered the screw heads. For this reason, tenting screws play an important role in ridge width gain and the improvement of grafting material's resorption. It is recommended that the heads of screws should be placed higher than the level of 
desired horizontal bone gain and over-graft performed in order to compensate for the resorption of the grafting materials [13].

Neither membrane exposure nor infection at the graft sites were recorded during eight months of follow-up because of the tension-free flap design and small sample size. Other authors have recorded different rates of infected complications, including one suppurative site [12], three infected sites [11] and 4.11\% with 35 treated sites [9]. The great amount of graft material placed, especially in severe ridge defects, may be responsible for membrane exposure, although the authors performed tension-free closures.

All tenting screws were successfully removed from the graft sites without fracturing the bone wall. Using a smooth surface without osteointegration, a small $1.5 \mathrm{~mm}$-body diameter and $6 \mathrm{~mm}$ or $8 \mathrm{~mm}$ length screws with 2.3-mm diameter heads were suitable not only for ridge shape construction but also reduced the inadvertent complications during screw removal, and frequency of screw-related flap exposure. We placed screws at an incline instead of perpendicular to the bony surface to obtain the ideal ridge form. Two tenting screws per defect site were used, one in the coronal position and one in the apical position. Other authors also placed additional screws every 4 to $6 \mathrm{~mm}$ to create an ideal ridge form. Placing supernumerary screws was thought not useful. Besides leading to inadequate space, using too few screws will limit reconstruction of the ideal alveolar ridge form.

Implants were inserted in all cases with good primary stability. In this study, Bio-Oss was used as a unique graft material without harvesting autogenous bone from secondary sites. This advantage reduced donor site morbidity. However, the healing time required was longer than what we anticipated at eight to 10 months [2]. A recent systematic review demonstrated that lateral ridge augmentation with Bio-Oss alone or in combination with autogenous bone graft seems to induce newly formed bone in histological assessment and increase the width of the alveolar process per clinical review. There was no significant difference between the two approaches [6].

This case series study had some limitations. Small sample sizes may affect several clinical aspects. The healing time was elongated due to the authors using ABBM alone. In the future time, the combination of xenograft materials and platelet-rich fibrin will be applied to shorten the healing time [14]. It is also necessary to conduct case series with larger sample sizes or randomized control trials to support the positive clinical results of the tent-pole technique.

\section{Conclusion}

Conducting the tent-pole technique employing native collagen membrane and ABBM is an effective method to improve the horizontal ridge dimension in defect sites, minimize postoperative complications, achieve better patient satisfaction, and support subsequent implant placement.

\section{Authors' Contributions}

TLD (D) 0000-0002-5495-1501 Conceptualization, Methodology, Investigation, Formal Analysis, Data Curation, Writing - Original Draft Preparation; Writing - Review and Editing and Visualization.

LDL (iD OOOO-0003-1 194-8991 Methodology, Validation, Investigation, Resources, Writing - Review and Editing; Supervision, Project Administration and Funding Acquisition.

All authors declare that they contributed to critical review of intellectual content and approval of the final version to be published. 


\section{Financial Support}

None.

\section{Conflict of Interest}

The authors declare no conflicts of interest.

\section{References}

[1] Tolstunov L. Classification of the alveolar ridge width: implant-driven treatment considerations for the horizontally deficient alveolar ridges. J Oral Implantol 2014; 40(Spec No):365-70. https://doi.org/10.1563/aaid-joi-D-14-00023

[2] Hämmerle CHF, Jung RE, Yaman D, Lang NP. Ridge augmentation by applying bioresorbable membranes and deproteinized bovine bone mineral: a report of twelve consecutive cases. Clin Oral Implants Res 2008; 19(1):19-25. https://doi.org/10.1111/j.1600-0501.2007.01407.x

[3] Blus C, Szmukler-Moncler S. Split-crest and immediate implant placement with ultra-sonic bone surgery: a 3-year life-table analysis with 230 treated sites. Clin Oral Implants Res 2006; 17(6):700-7. https://doi.org/10.1111/j.1600-0501.2006.01206.x

[4] Chiapasco M, Abati S, Romeo E, Vogel G. Clinical outcome of autogenous bone blocks or guided bone regeneration with e-PTFE membranes for the reconstruction of narrow edentulous ridges. Clin Oral Implants Res 1999; 10(4):278-88. https://doi.org/10.1034/j.1600-0501.1999.100404.x

[5] Yamauchi K, Takahashi T, Nogami S, Kataoka Y, Miyamoto I, Funaki K. Horizontal alveolar distraction osteogenesis for dental implant: long-term results. Clin Oral Implants Res 2013; 24(5):563-8. https://doi.org/10.1111/j.1600-0501.2011.02417.x

[6] Aludden HC, Mordenfeld A, Hallman M, Dahlin C, Jensen T. Lateral ridge augmentation with Bio-Oss alone or BioOss mixed with particulate autogenous bone graft: a systematic review. Int J Oral Maxillofac Surg 2017. 46(8):10301038. https://doi.org/10.1016/j.ijom.2017.03.008

[7] Le B, Rohrer MD, Prasad HS. Screw "tent-pole” grafting technique for reconstruction of large vertical alveolar ridge defects using human mineralized allograft for implant site preparation. J Oral Maxillofac Surg 2010; 68(2):428-35. https://doi.org/10.1016/j.joms.2009.04.059

[8] Di Stefano DA, Greco GB, Cinci L, Pieri L. Horizontal-guided bone regeneration using a titanium mesh and an equine bone graft. J Contemp Dent Pract 2015; 16(2):154-62. https://doi.org/10.5005/jp-journals-10024-1653

[9] Deeb GR, Tran D, Carrico CK, Block E, Laskin DM, Deeb JG. How effective is the tent screw pole technique compared to other forms of horizontal ridge augmentation? J Oral Maxillofac Surg 2017; 75(10):2093-8. https://doi.org/10.1016/j.joms.2017.05.037

[10] Bunyaratavej P, Wang HL. Collagen membranes: a review. J Periodontol 2001; 72(2):215-29. https://doi.org/10.1902/jop.2001.72.2.215

[11] Caldwell GR, Mills MP, Finlayson R, Mealey BL. Lateral alveolar ridge augmentation using tenting screws, acellular dermal matrix, and freeze-dried bone allograft alone or with particulate autogenous bone. Int J Periodontics Restorative Dent 2015; 35(1):75-83. https://doi.org/10.11607/prd.2260

[12] Urban IA, Nagursky H, Lozada JL, Nagy K. Horizontal ridge augmentation with a collagen membrane and a combination of particulated autogenous bone and anorganic bovine bone-derived mineral: a prospective case series in 25 patients. Int J Periodontics Restorative Dent 2013; 33(3):299-307. https://doi.org/10.1 1607/prd.1407

[13] Elnayef B, Porta C, Del Amo FSL, Mordini L, Gargallo-lbiol J, Hernández-Alfaro F. The fate of lateral ridge augmentation: a systematic review and meta-analysis. Int $\mathrm{J}$ Oral Maxillofac Implants 2018; 33(3):622-35. https://doi.org/10.11607/jomi.6290

[14] Tatullo M, Marrelli M, Cassetta M, Pacifici A, Stefanelli LV, Scacco S, et al. Platelet Rich Fibrin (P.R.F.) in reconstructive surgery of atrophied maxillary bones: clinical and histological evaluations. Int J Med Sci 2012; 9(10):872-80. https://doi.org/10.7150/ijms.5119 